\title{
ERG Rearrangement as a Clonal Expansion Marker for Prostate Cancer
}

\author{
Martin Braun, Roopika Menon, Pavel Nikolov and Sven Perner* \\ Institute of Pathology, Comprehensive Cancer Center, University Hospital of Tuebingen, Tuebingen, Germany
}

\begin{abstract}
Prostate cancer is the most commonly diagnosed neoplasia and the second most frequent cause of cancer specific death in men in the western civilization. The recent discovery and subsequent characterization of recurrent gene rearrangements of ETS genes - most frequently ERG - in the majority of prostate cancers is a milestone in translational prostate cancer research. In this review we summarize the latest findings on the $E R G$ rearrangement in prostate cancer. In particular, we focused on the relevance of the $E R G$ rearrangement as a marker to elucidate the heterogeneity of prostate cancer, a finding which until recently has been difficult to study. Furthermore, since prostate cancer is a multifocal disease in the majority of cases and it is still poorly understood which tumor focus is responsible for metastasis, we explicate the application of the $E R G$ rearrangement as a prostate cancer specific clonal expansion marker.
\end{abstract}

Keywords: ERG rearrangement, prostate cancer, gene fusion, clonal expansion marker.

\section{INTRODUCTION}

In men, prostate cancer $(\mathrm{PCa})$ is the most frequently observed malign neoplasia in the western world and the second most prevalent cause of cancer-specific death. Nevertheless, for the majority of PCa positive patients the cause of death is not the cancer itself [1]

To estimate the clinical course of PCa, patients PSAlevels, clinical tumor stage, the tumor volume and Gleasongrade in biopsy are commonly used parameters for stratification, which are of particular importance for making clinical decisions and predicting prognosis. However, these parameters are often insufficient for indisputably identifying patients who benefit from treatment. Consequently, it remains a challenge to distinguish between PCa with an aggressive or indolent clinical course. For the most part, this is due to the fact that the molecular biology of the cancerogenesis and progression of PCa is still not fully understood.

To dissect the heterogeneity of the clinical behavior and the molecular pathology of PCa, the development of reliable diagnostic, predictive and prognostic biomarkers is imperative. Promising objects of current PCa biomarker research are the recently identified recurrent gene fusions [2].

\section{THE TMPRSS2-ERG GENE FUSION-A FREQUENT EVENT IN PROSTATE CANCER}

The first discovered and best understood cancer-specific gene fusion is the Philadelphia-chromosome in chronic myelogenous leukemia (CML), resulting from a balanced translocation between chromosome 9 and 22 [3]. The translated fusion protein, a tyrosine kinase, which is constitutively active, is responsible for uninhibited proliferation of the tumor

\footnotetext{
*Address correspondence to this author at the Institute of Pathology,Comprehensive Cancer Center,University Hospital of Tuebingen Liebermeisterstr. 8 D-72076 Tuebingen Germany; Tel: +49 707129 84926; Fax: +49 707129 2258; E-mail: sven.perner@medizin.uni-tuebingen.de
}

cells. Based on the discovery of the Philadelphia chromosome, a targeted therapy has been developed, specifically inhibiting the activity of the tyrosine kinase and thus ameliorating prognosis of CML patients.

Recently, genetic rearrangements resulting in the continuous over expression of potential oncogenes have been found in approximately $50 \%$ of $\mathrm{PCa}$ in prostatectomy cohorts. The most frequent rearrangement involves the 5' region of the prostate-specific androgen regulated transmembrane protease serine 2 (TMPRSS2) and members of the erythroblast transformation-specific (ETS) family of transcription factors [2]. Of these, the most common fusion event is the fusion between the ETS member ERG and TMPRSS2. Since the first description of gene fusions in $\mathrm{PCa}$ by Tomlins et al. in 2005, a multitude of other gene fusions have been discovered, but occur at much lower frequencies [4]. In most cases, the TMPRSS2-ERG gene fusion is acquired through a deletion of the genetic material between $E R G$ and TMPRSS2, located on 21q. The other mechanism results from an insertion of the $E R G$ gene (Fig. 1) [5].

So far, the TMPRSS2-ERG fusion has been examined in nearly 40 studies with a total number of over 2200 cases. Independent reports conclude that about $50 \%$ of PCa samples harbor a fusion between TMPRSS2 and ERG in prostatectomy cohorts [4-16]. Interestingly, in incidentally diagnosed PCa the frequency is as low as 15 to $35 \%$. This may be due to smaller proportion of aggressive PCa cases amongst those $[17,18]$. Still, the true reason for the difference in the prevalence rate between prostatectomy cohorts and incidentally diagnosed cohorts remains unknown.

\section{METHODS FOR DETECTION OF THE TMPRSS2- ERG GENE FUSION}

There are different ways to detect the TMPRSS2-ERG fusion. On the transcript level, the fusion product can be identified by quantitative or non-quantitative polymerase 


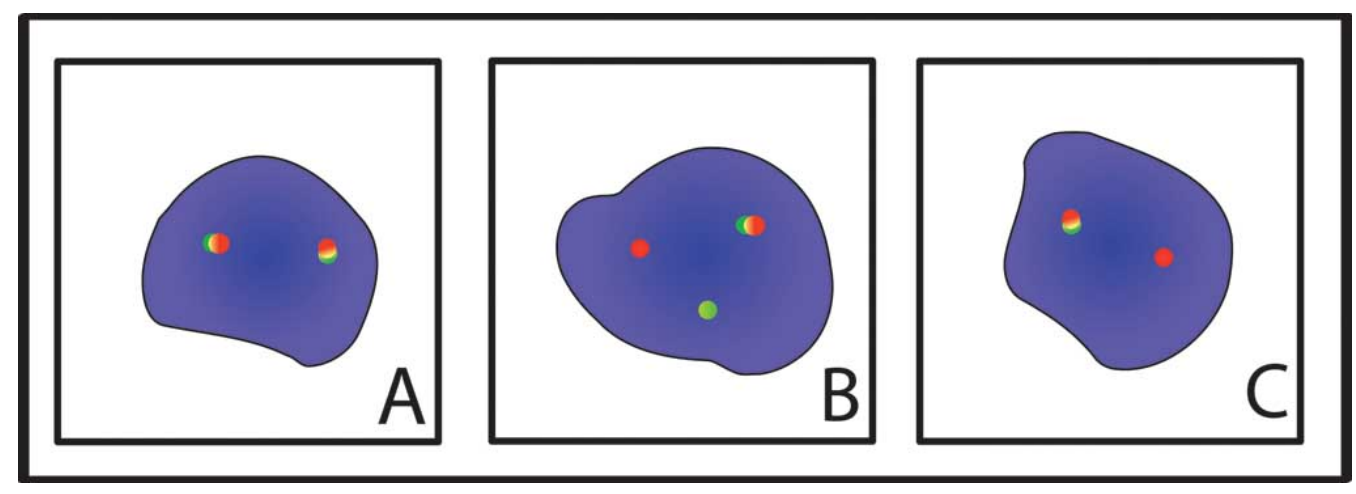

Fig. (1). Readout of the $E R G$ break-apart FISH assay. (A) Schematic nucleus without the $E R G$ rearrangement (wild type). A nucleus without the $E R G$ rearrangement shows two yellow signals which indicate the still juxtaposed, differentially labelled centromeric (red) and telomeric (green) regions of $E R G$. (B) Schematic nucleus with the ERG rearrangement through insertion of one allele. The separated red and green probes indicate the rearrangement of the $E R G$ locus. The non-rearranged allele is indicated by the yellow signal. (C) Schematic nucleus with the $E R G$ rearrangement through deletion of one allele. The disappearance of the green signal in one allele shows the deletion of the telomeric region of $E R G$. The non-rearranged allele is indicated by the yellow signal.

chain reaction (PCR) methodologies [14]. PCR-based detection of the fusion transcript in a patient's urine is an alternate method that can be used to identify a TMPRSS2-ERG fusion positive prostate cancer focus $[19,20]$. The major advantage of such an urine-based test is its high rate of specificity, its non-invasiveness, and its potential for use as a screening-test to detect aggressive forms of PCa. However, so far, most studies have used fluorescence in-situ hybridization (FISH) assays to detect cancers with the TMPRSS2-ERG fusion on the genomic level (Fig. 1) [2,5]. Although, due to the relatively short $3 \mathrm{Mb}$ distance between the genes $E R G$ and TMPRSS2 and the limited resolution of FISH assays, a direct detection of the gene fusion is not feasible. Probing the telomeric and centromeric regions of the ERG gene, FISHbased assays visualize the presence of an $E R G$ rearrangement, which is often considered equivalent to a TMPRSS2$E R G$ gene fusion, even though it can not identify the true 5, partner of $E R G$ [2]. Despite the shortcomings of FISH assays in this context, the possibility to assess tumors on a cell by cell basis is a great advantage as compared to PCR-based methods and provided important insight into the development, progression and heterogeneity of PCa. Soon after the introduction of FISH-based ERG break-apart assays, independent studies could demonstrate that the ERG rearrangement is specific to a subset of high-grade prostatic intraepithelial neoplasias (PIN) and PCa foci, but does not occur in any kind of benign lesion, even not in closest proximity to neoplastic tissue with $E R G$ rearrangement (Fig. 2A) [12, 16]. Furthermore, the FISH assay could prove that each focus in a multifocal prostate cancer harbors an individual $E R G$ rearrangement status. Interestingly, each single cell within a specific tumor focus is characterized by the same rearrangement status [21-23].

\section{ASSESSING ERG REARRANGEMENT STATUS TO STUDY THE PROGRESSION FROM PIN TO INVASIVE CARCINOMA}

PIN is a commonly observed lesion in prostatectomy specimen and prostate biopsies. Several studies have shown that invasive PCa and PIN share some molecular features, but there is no clear evidence of PIN being a true precursor lesion of invasive carcinoma [24]. Therefore, recent studies have assessed the prevalence of $E R G$ rearrangement in the PIN lesions [9, 12, 16]. Approximately $20 \%$ of PIN lesions that are in close proximity to an $E R G$ rearranged invasive $\mathrm{PCa}$ are also positive for the $E R G$ rearrangement $[12,16]$. However, the majority of these cases lack the $E R G$ rearrangement in PIN, even though the adjacent PCa focus harbors the gene fusion. Importantly, if an ERG rearranged PIN lesion is detected in a biopsy, this is evidence for an $E R G$ rearranged invasive $\mathrm{PCa}$ focus within the same prostate [12, 16] (Fig. 2A). Together, these findings could be translated into contemporary clinical practice. If exclusively an $E R G$ rearranged PIN lesion is detected by prostate biopsies, but the biopsies do not contain any invasive $\mathrm{PCa}$ focus, $\mathrm{PCa}$ therapy might be considered without performing further biopsies, based on the fact that an $E R G$ rearranged PIN lesion proves the existence of an $E R G$ rearranged $\mathrm{PCa}$ focus within this prostate. Furthermore, these results support the hypothesis that at least subgroups of PIN lesions are true precursors of invasive PCa. On the other hand, it also suggests that most invasive PCa might not originate from PIN.

In a murine model, Klesovitch et al. assessed for the presence of a causal relationship between ERG expression and development/progression of PIN lesions. Notably, ERG over expression resulted in initiation of PIN lesions in this model. A displacement of basal epithelial cells with luminal epithelia cells was observed in these lesions. Subsequently, the luminal cells established direct contact with the stroma cell compartment. The loss of basal cells is known to be a critical milestone in prostate cancerogenesis [25]. Additional PI3K pathway activation further enhanced the initiation of these PIN lesions, King et al. [26]. Likewise, Carver et al. discovered a correlation between ERG over expression in PIN lesions and the loss of function of the tumor suppressor gene PTEN. Remarkably, 93\% of fusion positive PIN lesions showed low or no PTEN expression. Studies with murine prostate tissue demonstrated that the $E R G$ rearrangement and the reduced PTEN expression in PIN lesions were associated with a higher incidence of $\mathrm{PCa}$ and an increased progression to invasive PCa. An even increased incidence was observed, if the expression of PTEN was significantly reduced in PIN [27]. Together, these results suggest that the $E R G$ rearrangement is not an independent event in PCa. This rear- 
rangement cooperates with other genetic aberrations, such as PTEN loss of function, enhancing cancerogenesis and tumor progression.

\section{ASSESSING ERG REARRANGEMENT STATUS TO STUDY THE PROGRESSION FROM CONVEN- TIONAL ACINAR PCA TO SMALL CELL PCA}

Small cell cancer of the prostate (SCPC) is a rare but aggressive disease with poor prognosis even if detected in a localized stage. It is poorly understood and controversially discussed as to whether SCPC is an independent tumor entity or just the phenotype of a dedifferentiated acinar PCa [28, 29]. Recently, Scheble et al. assessed a cohort of 15 SCPC for the $E R G$ rearrangement [30]. In agreement with Han et al. they found that the vast majority of SCPC (83\%) harbor the ERG rearrangement [31]. Remarkably, if samples contained a transition from conventional acinar PCa into SCPC, both phenotypes harboured the $E R G$ rearrangement (Fig. 2B). Also, the ERG rearrangement has not been found in any cancer of epithelial origin but $\mathrm{PCa}$ [32]. Together, these findings suggest that SCPC is related to common acinar $\mathrm{PCa}$ and can be seen as a dedifferentiated phenotype of $\mathrm{PCa}$ with one common cell of origin.

\section{ASSESSING ERG REARRANGEMENT STATUS TO IDENTIFY AGGRESSIVE PCA}

Shortly after discovering the $E R G$ rearrangement in $\mathrm{PCa}$, Demichelis et al. observed in a watchful waiting cohort of

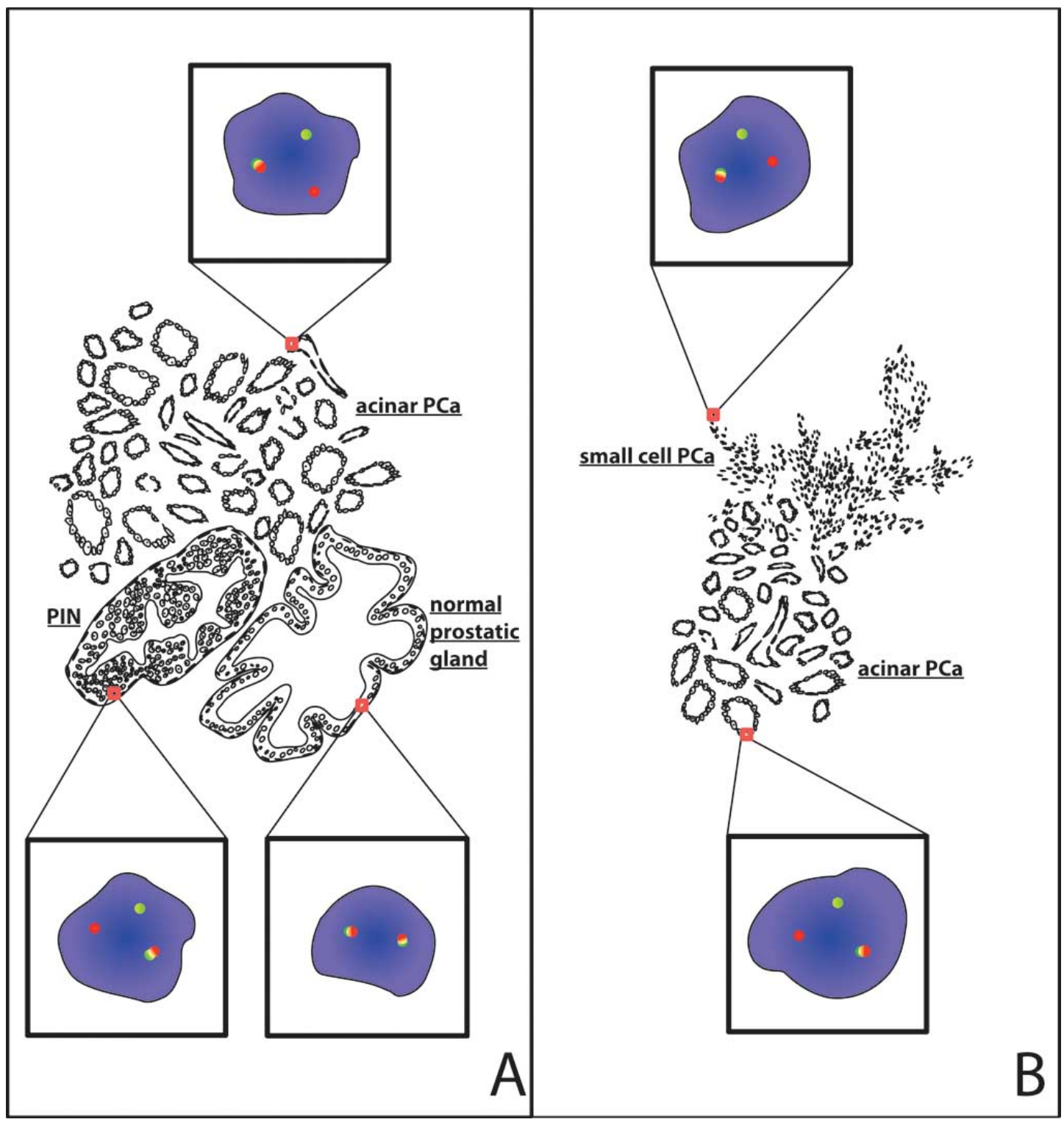

Fig. (2). (A). Schematic of a normal prostatic gland, a PIN lesion, and adjacent acinar PCa with corresponding $E R G$ rearrangement status. The normal prostatic gland (lower right) is not ERG rearranged (illustrated by magnified representative FISH nucleus). The PIN lesion (lower left) and the adjacent acinar PCa (upper part) both harbor the $E R G$ rearrangement (illustrated by magnified representative FISH nuclei). (B) Schematic of a conventional acinar PCa transitioning into small cell PCa with corresponding ERG rearrangement status. Both, the acinar PCa (lower part) and small cell PCa (upper part) harbor the ERG rearrangement (illustrated by magnified representative FISH nuclei). 
incidentally diagnosed PCa patients, that patients harboring the $E R G$ rearrangement have a significantly increased prostate cancer-specific death rate [17]. Attard et al. confirmed these results on a similarly designed cohort [18]. About $90 \%$ of patients negative for the gene rearrangement survived at least 8 years, whereas those positive for the rearrangement had a statistically significant decreased actuarial survival rate. Amongst those, a subgroup of patients with a duplication of $E R G$ gene faced a worse outcome: only $25 \%$ of these patients lived longer than 8 years after initial diagnosis. Also, Nam et al. found on a prostatectomy cohort that the patients with a gene rearrangement had a significantly increased risk of disease recurrence [11]. In addition, Perner et al. showed evidence that the clinical course could also depend on the $E R G$ rearrangement mechanism. ERG rearrangement, through deletion, is associated with higher tumor stage, an increased trend for PSA recurrence, and higher frequency of metastasizing to the pelvic lymph nodes [5]. Observing that all of the assessed metastatic PCa sites containing the $E R G$ rearrangement showed fusion through deletion, Mehra et al. have supported this hypothesis [33].

Controversially, other studies reported an association of the gene rearrangement with clinical features of better prognosis [34, 35]. In cases of clinically localized surgically treated prostate cancers, Gopalan et al. reported an association of ERG rearrangement with lower grade, but no association with stage, biochemical recurrence, metastases, or death [36].

Still, prospective studies are needed to further explore the prognostic relevance of the $E R G$ rearranged $\mathrm{PCa}$ on well defined cohorts.

\section{ASSESSING ERG REARRANGEMENT STATUS TO STUDY THE PROGRESSION OF MULTIFOCAL PCA AND METASTATIC DISSEMINATION}

In most of the cases, PCa is a multifocal disease with multiple tumor foci arising independently from each other [37]. Noteworthy, morphology, Gleason grade, and size of these different tumor foci can be prevalently heterogenic. A problem in clinical practice is that a needle biopsy often fails to capture all of the foci and may miss the most aggressive tumor focus. Several studies have assessed all foci of multifocal PCa for $E R G$ rearrangement status [21-23]. They have discovered that $\mathrm{PCa}$ heterogeneity is also reflected by the $E R G$ rearrangement. I.e., if there are several tumor foci in a prostatectomy sample, each of these harbor an individual fusion status. Interestingly, it was not always the largest tumor focus or the focus with the highest Gleason Grade which harbored the ERG rearrangement (Fig. 3) [38]. Remarkably, the rearrangement status within a specific tumor focus is homogenous on a cell by cell basis. These findings strongly support the hypothesis that multifocal PCa is a heterogeneous disease with each tumor focus deriving from an independent cell of origin.

Moreover, this knowledge could be applied to clinical routine. Since a biopsy does not systematically capture all tumor foci, it is possible that it fails to sample the most aggressive tumor focus. For that reason, a subsequent urine test for the fusion transcript would be helpful to detect missing tumor foci which are missed by biopsies.

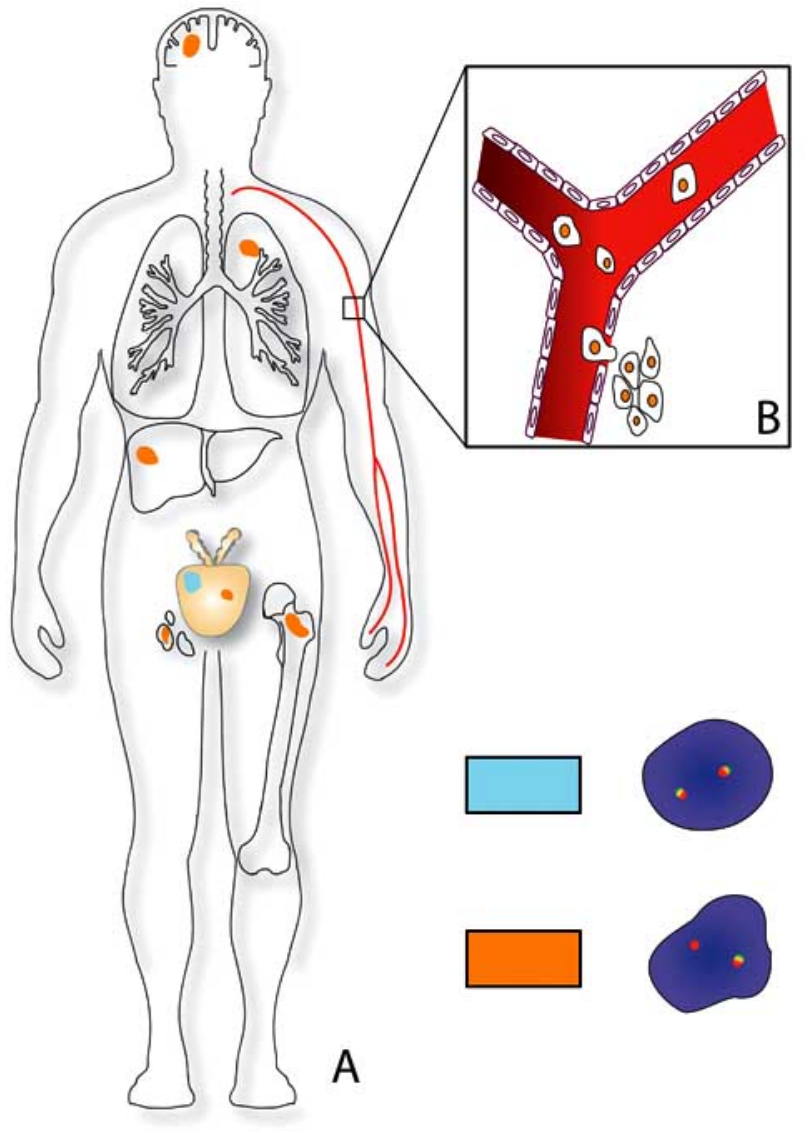

Fig. (3). (A). Schematic human with a mulitfocal PCa in the prostate, and $\mathrm{PCa}$ metastases in the inguinal lymph nodes, the left femur, the liver, the apex of the left lung, and the brain with corresponding $E R G$ rearrangement status. The prostate contains two $\mathrm{PCa}$ foci (orange and blue areas). The tumor focus (marked blue) with the largest volume is not $E R G$ rearranged. The metastasizing, smaller, lower Gleason grade tumor focus (marked orange) harbors the $E R G$ rearrangement. All the lymph node and distant metastases as well as the circulating tumor cells are uniformly positive for the $E R G$ rearrangement. (B) Circulating tumor cells in a schematic blood vessel.

\section{ERG REARRANGEMENT AS A CLONAL MARKER OF EXPANSION FOR PCA}

From the seeding PCa focus cells are spreading into lymph nodes and/or distant organ sites. Circulating tumor cells (CTCs) - cells that have detached from a tumor focus and have gained access to the angiolymphatic system- might give rise to the subsequent growth of metastases (Fig. 3). However, it is an ongoing debate, which tumor focus gives rise to metastasis. Often it is assumed that the focus with highest Gleason score or largest tumor volume is metastasizing. But until now, there is no clear evidence for this hypothesis. Commonly used markers like androgen receptor, PTEN, or PSA are inconsistently expressed during metastatic progression, and thus not capable of identifying the originating focus. Even histomorphologic features like Gleason grade might change during progression [39]. Studying the $E R G$ rearrangement status as a marker for clonal expansion gave insight into this issue. As mentioned above, all tumor 
cells belonging to a specific focus are homogenous for the ERG rearrangement status. Perner et al. observed in cases with multifocal primary $\mathrm{PCa}$ and matched lymph nodes metastasis, that the lymph node metastasis and at least one primary PCa focus was characterized by the same $E R G$ rearrangement status $[5,38]$. This underlines that a seeding $\mathrm{PCa}$ focus is not necessarily the one with highest Gleason grade or the largest tumor volume, but the one harboring the $E R G$ rearrangement (Fig. 3). Assessing 24 cases of hormone independent metastatic PCa, Mehra et al. discovered that distant metastases at multiple sites all harbored the same rearrangement status [33]. In addition, Attard et al. showed that a primary PCa focus and the corresponding isolated CTCs were uniformly characterized by the same ERG rearrangement status - in contrast to a significant heterogeneity of AR and PTEN copy number changes [39].

In conclusion, all of the assessed primary PCa foci and corresponding metastases (regional lymph node, distant metastases, and CTCs) showed homogeneity with regard to the individual $E R G$ rearrangement status. The $E R G$ rearrangement status seems to be a clonal expansion marker that is capable of identifying the seeding tumor focus. The influence of identifying the seeding tumor focus is still underestimated in today's clinical practice, but may show to have a promising impact soon. Especially with regard to translational research, it will be a huge advantage to be able to identify the metastasizing focus, e.g. to comprehensively study the biology of the seeding focus in order to develop new clinical therapies and biomarkers.

\section{CONCLUSION}

The recent discovery and detailed characterization of recurrent gene rearrangements in $\mathrm{PCa}$ is a milestone in translational $\mathrm{PCa}$ research. A multitude of studies have been working on deciphering the relationship between these gene rearrangements and the clinical heterogeneity of PCa progression. Even though our understanding of these events have been emerging, it is still poorly understood, how exactly these aberration have an effect on this disease.

\section{DISCLOSURES}

The Brigham and Women's Hospital and the Universitiy of Michigan have filed a patent on ETS gene rearrangements in prostate cancer, on which Perner is a coinventor, and the diagnostic field of use has been licensed to Gen-Probe Inc. Gen-Probe has not played a role in the preparation, review, or approval of the article.

\section{ACKNOWLEDGEMENT}

This work was supported by a grant of the German Research Foundation (Deutsche Forschungsgemeinschaft, DFG, Emmy-Noether-Program, PE1179/2-1) and the University Hospital of Tuebingen (fortuene Program, No. 1809$1-0)$ to S.P.

\section{REFERENCES}

[1] Jemal A, Siegel R, Ward E, Hao Y, Xu J, Thun MJ. Cancer statistics, 2009. CA Cancer J Clin 2009; 59(4): 225-49.

[2] Tomlins SA, Rhodes DR, Perner S, et al. Recurrent fusion of TMPRSS2 and ETS transcription factor genes in prostate cancer. Science 2005; 310(5748): 644-8.
[3] Deininger M, Buchdunger E, Druker BJ. The development of imatinib as a therapeutic agent for chronic myeloid leukemia. Blood 2005; 105(7): 2640-53.

[4] Tomlins SA, Bjartell A, Chinnaiyan AM, et al. ETS gene fusions in prostate cancer: from discovery to daily clinical practice. Eur Urol 2009; 56(2): 275-86.

[5] Perner S, Demichelis F, Beroukhim R, et al. TMPRSS2:ERG fusion-associated deletions provide insight into the heterogeneity of prostate cancer. Cancer Res 2006; 66(17): 8337-41.

[6] Kumar-Sinha C, Tomlins SA, Chinnaiyan AM. Recurrent gene fusions in prostate cancer. Nat Rev Cancer 2008; 8: 497-511.

[7] Iljin $\mathrm{K}$, Wolf $\mathrm{M}$, Edgren $\mathrm{H}$, et al. TMPRSS2 Fusions with oncogenic ETS factors in prostate cancer involve unbalanced genomic rearrangements and are associated with HDAC1 and epigenetic reprogramming. Cancer Res 2006; 66(21): 10242-6.

[8] Mehra R, Tomlins SA, Shen R, Nadeem O, et al. Comprehensive assessment of TMPRSS2 and ETS family gene aberrations in clinically localized prostate cancer. Mod Pathol 2007; 20(5): 538-44.

[9] Cerveira N, Ribeiro FR, Peixoto A, et al. TMPRSS2-ERG gene fusion causing ERG overexpression precedes chromosome copy number changes in prostate carcinomas and paired HGPIN lesions. Neoplasia 2006; 8(10): 826-32.

[10] Clark J, Attard G, Jhavar S, et al. Complex patterns of ETS gene alteration arise during cancer development in the human prostate. Oncogene 2008; 27(14): 1993-2003.

[11] Nam RK, Sugar L, Yang W, et al. Expression of the TMPRSS2:ERG fusion gene predicts cancer recurrence after surgery for localised prostate cancer. Br J Cancer 2007; 97(12): 16905 .

[12] Perner S, Mosquera JM, Demichelis F, et al. TMPRSS2-ERG fusion prostate cancer: an early molecular event associated with invasion. Am J Surg Pathol 2007; 31(6): 882-8.

[13] Soller MJ, Isaksson M, Elfving P, Soller W, Lundgren R, Panagopoulos I. Confirmation of the high frequency of the TMPRSS2/ERG fusion gene in prostate cancer. Genes Chromosomes Cancer 2006; 45(7): 717-9.

[14] Wang J, Cai Y, Ren C, Ittmann M. Expression of variant TMPRSS2/ERG fusion messenger RNAs is associated with aggressive prostate cancer. Cancer Res 2006; 66(17): 8347-51.

[15] Yoshimoto M, Joshua AM, Chilton-Macneill S, et al. Threecolor FISH analysis of TMPRSS2/ERG fusions in prostate cancer indicates that genomic microdeletion of chromosome 21 is associated with rearrangement. Neoplasia 2006; 8(6): 465-9.

[16] Mosquera JM, Perner S, Genega EM, et al. Characterization of TMPRSS2-ERG fusion high-grade prostatic intraepithelial neoplasia and potential clinical implications. Clin Cancer Res 2008; 14(11): 3380-5.

[17] Demichelis F, Fall K, Perner S, et al. TMPRSS2:ERG gene fusion associated with lethal prostate cancer in a watchful waiting cohort. Oncogene 2007; 26(31): 4596-9.

[18] Attard G, Clark J, Ambroisine L, et al. Duplication of the fusion of TMPRSS2 to ERG sequences identifies fatal human prostate cancer. Oncogene 2008; 27(3): 253-63.

[19] Laxman B, Tomlins SA, Mehra R, et al. Noninvasive detection of TMPRSS2:ERG fusion transcripts in the urine of men with prostate cancer. Neoplasia 2006; 8(10): 885-8.

[20] Hessels D, Smit FP, Verhaegh GW, Witjes JA, Cornel EB, Schalken JA. Detection of TMPRSS2-ERG fusion transcripts and prostate cancer antigen 3 in urinary sediments may improve diagnosis of prostate cancer. Clin Cancer Res 2007; 13(17): 5103-8.

[21] Mehra R, Han B, Tomlins SA, et al. Heterogeneity of TMPRSS2 gene rearrangements in multifocal prostate adenocarcinoma: molecular evidence for an independent group of diseases. Cancer Res 2007; 67(17): 7991-5.

[22] Barry M, Perner S, Demichelis F, Rubin MA. TMPRSS2-ERG fusion heterogeneity in multifocal prostate cancer: clinical and biologic implications. Urology 2007; 70(4): 630-3.

[23] Furusato B, Gao CL, Ravindranath L, et al. Mapping of TMPRSS2-ERG fusions in the context of multi-focal prostate cancer. Mod Pathol 2008; 21(2): 67-75.

[24] DeMarzo AM, Nelson WG, Isaacs WB, Epstein JI. Pathological and molecular aspects of prostate cancer. Lancet 2003; 361(9361): 955-64.

[25] Klezovitch O, Risk M, Coleman I, et al. A causal role for ERG in neoplastic transformation of prostate epithelium. Proc Natl Acad Sci U S A 2008; 105(6): 2105-10. 
[26] Clark JP, Cooper CS. ETS gene fusions in prostate cancer. Nat Rev Urol 2009; 6(8): 429-39.

[27] Carver BS, Tran J, Gopalan A, et al. Aberrant ERG expression cooperates with loss of PTEN to promote cancer progression in the prostate. Nat Genet 2009; 41(5): 619-24.

[28] Hansel DE, Nakayama M, Luo J, Abukhdeir AM, et al. Shared TP53 gene mutation in morphologically and phenotypically distinct concurrent primary small cell neuroendocrine carcinoma and adenocarcinoma of the prostate. Prostate 2009; 69(6): 603-9.

[29] Spiess PE, Pettaway CA, Vakar-Lopez F, et al. Treatment outcomes of small cell carcinoma of the prostate: a single-center study. Cancer 2007; 110(8): 1729-37.

[30] Scheble VJ, Braun M, Wilbertz T, et al. ERG rearrangement in small cell prostate and lung cancer. Histopathol 2010; in press.

[31] Han B, Mehra R, Suleman K, et al. Characterization of ETS gene aberrations in select histologic variants of prostate carcinoma. Mod Pathol 2009; 22(9): 1176-85.

[32] Scheble VJ, Braun M, Ruiz C, et al. ERG rearrangement is specific to prostate cancer and does not occur in any other common epithelial tumour. Mod Pathol 2010; in press.

[33] Mehra R, Tomlins SA, Yu J, et al. Characterization of TMPRSS2ETS gene aberrations in androgen-independent metastatic prostate cancer. Cancer Res 2008; 68(10): 3584-90.
[34] Saramaki OR, Harjula AE, Martikainen PM, Vessella RL, Tammela TL, Visakorpi T. TMPRSS2:ERG fusion identifies a subgroup of prostate cancers with a favorable prognosis. Clin Cancer Res 2008; 14(11): 3395-400.

[35] Darnel AD, Lafargue CJ, Vollmer RT, Corcos J, Bismar TA. TMPRSS2-ERG fusion is frequently observed in Gleason pattern 3 prostate cancer in a Canadian cohort. Cancer Biol Ther 2009; 8(2): $125-30$

[36] Gopalan A, Leversha MA, Satagopan JM, et al. TMPRSS2-ERG gene fusion is not associated with outcome in patients treated by prostatectomy. Cancer Res 2009; 69(4): 1400-6.

[37] Chen ME, Johnston DA, Tang K, Babaian RJ, Troncoso P. Detailed mapping of prostate carcinoma foci: biopsy strategy implications. Cancer 2000; 89(8): 1800-9.

[38] Perner S, Svensson M, Hossain R, et al. ERG rearrangement metastasis patterns in locally advanced prostate cancer. Urology 2010; in press.

[39] Attard G, Swennenhuis JF, Olmos D, et al. Characterization of ERG, AR and PTEN gene status in circulating tumor cells from patients with castration-resistant prostate cancer. Cancer Res 2009; 69(7): 2912-8.

(C) Braun et al.; Licensee Bentham Open.

This is an open access article licensed under the terms of the Creative Commons Attribution Non-Commercial License (http://creativecommons.org/licenses/ by-nc/3.0/) which permits unrestricted, non-commercial use, distribution and reproduction in any medium, provided the work is properly cited. 